\title{
HÁBITOS DE ESTUDIO Y RENDIMIENTO ACADÉMICO EN LOS ESTUDIANTES DE INGENIERÍA MECÁNICA
}

\section{HABITS OF STUDY AND ACADEMIC PERFORMANCE IN MECHANICAL ENGINEERING STUDENTS}

\author{
MSc. Eduar Bayona Ibáñez ${ }^{1}$, MSc. Isbelia Karina Ricon Parada ${ }^{2}$ \\ Universidad Francisco de Paula Santander, Ocaña, Colombia, Facultad de Ingeniería, \\ Grupo de Investigación GRUCITE \\ Sede Algodonal, Ocaña, Norte de Santander, Colombia \\ ebayonai@ufpso.edu.co,ikrinconp@ufpso.edu.co
}

(+57) (7) 5690088

\begin{abstract}
Resumen: Los factores que influyen en el rendimiento académico de los estudiantes son diversos, en la investigación se evaluó que tanto los estudiantes conocen su oficio y se determinó si existe correlación entre los hábitos de estudio y el rendimiento académico del programa de Ingeniería Mecánica de la Universidad Francisco de Paula Santander, Ocaña. Se recopilaron investigaciones a nivel nacional e internacional, encontrando que son muy pocas las investigaciones sobre el tema de hábitos de estudio y el rendimiento académico, tal como lo afirmó Martínez y Torres (2009) debido al error de considerar que los estudiantes cuando ingresan a la universidad ya cuentan con hábitos de estudio bien estructurados.

Para determinar el grado en el que los estudiantes conocen su oficio se aplicó la prueba de Pozar (2014) Inventario de Hábitos de Estudio (IHE), se realizaron dos mediciones, una en el 2014 y la otra en el 2016.
\end{abstract}

Palabras clave Hábitos de Estudio, Métodos de Estudio, Rendimiento Académico, Pozar.

\begin{abstract}
Factors influencing students' academic performance are diverse, in the research it was evaluated that both students know their trade and it was determined if there is correlation between study habits and academic performance of the program of Mechanical Engineering of the Francisco Paula Santander, Ocaña University . Research was collected at the national and international levels, finding that there is very little research on the subject of study habits and academic performance, as Martínez and Torres (2009) affirmed because of the error of considering that students when they enter University students already have well structured study habits.

In order to determine the degree to which the students know their occupation, the Pozar (2014) Test of Study Habits (IHE) test was applied, two measurements were taken, one in 2014 and the other in 2016.
\end{abstract}

Keywords: Study Habits, Study Methods, Academic Performance, Pozar.

\section{INTRODUCCIÓN}

Los diferentes cambios que desde inicio de este milenio han acompañado a la educación superior: condiciones de calidad, registro calificado procesos de certificación y acreditación, créditos académicos, flexibilidad curricular, entre otros, resaltan la importancia del autoaprendizaje, trabajo independiente de los estudiantes en su proceso de 
formación, se modifican no solo las dinámicas institucionales en el proceso educativo, sino tambien las dinámicas de aprendizaje de los estudiantes. Lo anterior, hace que los sujetos aborden nuevos hábitos y procedimientos en el la forma de estudiar; que trasciendan la costumbre de memorizar una serie de datos de un determinado texto o de una explicación o información impartida por el docente.

Estudiar como responsabilidad fundamental (pero no única) del estudiante en su proceso de formación, requiere de un método de estudio que le permita de forma lógica, gradual y sistemática enfrentar la información, conocimientos que le permita aprender, comprendiendo lo que enfrenta en su estudio. Martínez (2007) señala que no todos los estudiantes hacen frente con éxito a los nuevos desafíos que la Universidad plantea: aumento de la exigencia, necesidad creciente de organización del trabajo académico, desarrollo de procesos cognitivos y metacognitivos, mayor dedicación al estudio, autonomía. La definición, apropiación y puesta en acción de un método de estudio por parte de estudiante en su proceso de formación, requiere de la definición de hábitos de estudio que le permitan abordar su propio método de estudio, enfrentar su desempeño académico y su aprendizaje. Hernández manifiesta que los hábitos se empiezan a establecer desde los siete u ocho años y que estos dependen de otros hábitos como la concentración, el orden y la atención. Señala que el sujeto que ha crecido respetando límites, rutinas y hábitos (sueño, alimentación e higiene) no tendrá dificultad para adquirir el hábito de estudiar.

Ortega (2012) cita en su trabajo de investigación, los autores que se presentan a continuación, los cuales brindan un panorama de conceptos sobre los hábitos de estudio: Poves (2001) señala que el hábito de estudio es una acción que se realiza todos los días aproximadamente a la misma hora, la reiteración de ésta conducta en el tiempo va generando un mecanismo inconsciente; para Hernández (1988) son un conjunto de hábitos de trabajo intelectual que afectan a las funciones de motivación, condiciones físicas y destrezas instrumentales básicas para el estudio; cada una de éstas proporciona elementos que permiten un adecuado desenvolvimiento del estudiante en el que hacer educativo, así como en su contexto personal. Martínez y Torres (2009) definen a los hábitos de estudio como la práctica constante de las mismas actividades; se requiere de acciones cotidianas, las cuales serán con el tiempo un hábito afectivo siempre y cuando sean asumidas con responsabilidad, disciplina y orden. Belaunde
(1994) considera que el concepto de hábito de estudio está referido al modo como el individuo se enfrenta cotidianamente a su quehacer educativo, es la costumbre natural de procurar aprender permanentemente, lo cual implica la forma en el que el individuo se organiza en cuanto a tiempo, espacio, técnica y métodos, concretos que utiliza para estudiar". Como se puede apreciar la repetición de la conducta dirigida al aprendizaje, enmarcada en unas condiciones que direccionen la conducta al éxito escolar, caracterizan la definición de hábito d estudio.

Otra variable objeto de estudio en la presente investigación fue el rendimiento académico, el cual es definido por Villarroel (1989), como las calificaciones que obtuvo el estudiante por el aprendizaje adquirido en una asignatura, materia o curso. Pizarro (1985), señala que el rendimiento académico es un indicador del éxito frente a las demandas de la formación docente, es una medida de las capacidades respondientes en forma estimativa, es decir lo que una persona ha aprendido como consecuencia de un proceso de formación. Desde la perspectiva del estudiante, el rendimiento académico se define como la capacidad respondiente del alumno frente a estímulos educativos, la cual es susceptible de ser interpretada según propósitos establecidos. Atendiendo a lo anterior, se asumió en el estudio el rendimiento académico valorado desde el promedio ponderado del estudiante en el semestre académico.

Ha permitido analizar la relación entre hábitos de estudio y rendimiento académico es un elemento importante en el contexto. universitaria, ante la necesidad de trabajo independiente que el estudiante debe realizar para cumplir con los objetivos de los diferentes programas de asignaturas de una carrera universitaria, además porque los resultados de la investigación brindan información objetiva sobre los hábitos de estudio de los estudiantes y sobre su propio método de estudio, lo cual permitirá no solo a la institución sino también a los docentes tomar decisiones que posibiliten la calidad y mejoramiento académico de los estudiantes.

Para dar cumplimiento a los objetivos de la investigación, se aborda la Institución de Educación Superior, la Universidad Francisco de Paula Santander, Ocaña, del Municipio de Ocaña, departamento Norte de Santander en la que se ofertan programas académicos en las áreas: Administrativas, Agrarias, Ingenierías, Educación y Artes. La población se encuentra conformada por 
los estudiantes de primer semestre, año 2014 del programa de Ingeniería Mecánica.

Estudiantes que conformaron la muestra en las dos mediciones:

Tabla 1, Estudiantes programa de Ingeniería

Mecánica

\begin{tabular}{llc}
\hline \multirow{2}{*}{ Programa de Ingeniería de Mecánica } & \multicolumn{2}{l}{ Numero de Estudiantes } \\
\cline { 2 - 3 } & Medición 1 & Medición 2 \\
\cline { 2 - 3 } & 78 & 43 \\
\hline
\end{tabular}

Fuente. Elaboración Propia

\section{RECOMENDACIONES}

La carencia de investigaciones relacionadas con los hábitos de estudio en la educación superior permite seguir trabajando, tal como lo manifestó (Martínez y Torres 2009; Castellanos et el, 2011) quienes fueron los primeros en mostrar la importancia de hacer investigaciones que se orienten a determinar las causas que afectan al rendimiento académico, que le permitan a las Instituciones tomar decisiones oportunas y se encaminen a la calidad efectiva, se utiliza la base de datos recolectadas por el grupo de investigación (Caballero A. et al., 2013).

Luego de realizar diferentes estudios para conocer la existencia de las investigaciones que se relaciones con el rendimiento académico y los hábitos de estudio, se pueden nombrar las investigaciones de (Martínez y Torres 2009),Escalante, (Linzaga y Merlos 2008), (Villegas y Muñoz 2009), (Villota, 2013), (Ríos y Ramos, 2013), (Torres et el, 2009), (Álvarez y Fernández, 2013) y Pozar(2014), determinaron que no son suficientes, debido a la multitud de cambios y oportunidades que se han generado en su mayor parte gracias a la tecnología, debido a lo complejo del problema el instrumento de Pozar (2014) contribuye "un primer paso de aproximación al problema". Ya que tiene como objetivo es "abrir brecha, definiendo las actitudes del estudiante hacia su trabajo. La tarea esencial, la importante y verdaderamente orientadora, viene después cuando el educador interviene sobre estos aspectos".

El nuevo interrogante que se genera en cuando a las investigaciones de Pozar (2014), tiene que ver con la funcionalidad de los baremos, aunque el autor argumenta que no existían diferencias suficientemente relevantes que justificaran una nueva baremación y que el uso de los baremos ya existentes permitía una buena evaluación de los hábitos de estudio en los escolares", es claro que algo se tiene que hacer, pues en la presente investigación se determinó que no existe relación entre el rendimiento académico y los hábitos de estudio.

El aporte de la investigación permite hacer un acercamiento, conocer cómo es que los estudiantes desempeñan su responsabilidad con académica, las técnicas y métodos que más usan y la efectividad o no en sus resultados.

Mediante la aplicación la prueba de Pozar (2014) IHE a estudiantes de la Facultad de Ingenierías, de la Universidad Francisco de Paula Santander, Ocaña, en las dos mediciones, la primera realizada cuando inician su vida universitaria y la segunda cuando ha trascurrido un año. El perfil muestra que el grupo en general presenta un resultado global normal, es decir muy poco o casi nada en la aplicación de hábitos de estudio, encontrando que existen aspectos que se deben mejorar en cada una de las escalas, para el caso de las condiciones ambientales, la calificación es buena bajo, indicando que el grupo en cuanto a las condiciones físicas, personales, comportamiento académico y rendimiento, cumple satisfactoriamente; por lo tanto se debe mantener o mejorar, para ello, se recomienda leer y aplicar la herramienta "Aprender a Estudiar" de Pozar (2014).

En la Planificación del Estudio, la clasificación es normal; indicando que en tanto en la Organización como en Horarios se cumple satisfactoriamente; para mejorar los resultados se recomienda leer y aplicar la herramienta "Aprender a Estudiar" de Pozar (2014). En el Empleo de los Materiales se obtuvo una calificación Normal en lectura, Libros y Resúmenes, se recomienda una lectura a la herramienta "Didáctica del Estudio". En la escala de Asimilación de Contenidos, se cumple satisfactoriamente, pero es necesario mantener los resultados o mejorarlos, se recomienda leer la "Didáctica del Estudio", servirá para el perfeccionamiento de los métodos aplicados. Para la Asimilación de Contenidos la calificación es Normal para los aspectos de memorización, trabajo individual $\mathrm{y}$ en equipo, se debe mantener o mejorar, mediante la aplicación de las normas “Aprender a Estudiar" de Pozar (2014).

La calificación normal de la categoría de Sinceridad, garantiza que las respuestas de cada uno de los estudiantes han sido bastante sinceras. "Con este diagnóstico se puede emprender una "campaña" de mejora de los hábitos de trabajo y 
estudio proporcionando a los alumnos las normas concretas de actuación” (Pozar F. , 2014, pág. 27).

Los estudiantes cuentan con conocimiento y aplicación de las técnicas de estudio, pero no lo hacen con rigurosidad necesaria, en algunos casos lo aplican previamente para presentar un examen, sin que se genere hábito, siendo el bajo rendimiento académico una consecuencia del desconocimiento de un adecuado manejo de métodos y técnicas de estudio en concordancia con lo manifestado por (Villegas y Muñoz, 2009).

El inventario de Hábitos de Estudio de (Pozar, 2014), permitió la evaluación de los hábitos de estudio que influyen en las actividades académicas de los estudiantes del programa de Ingeniería mecánica adscritos a la Facultad de Ingeniería de la Universidad Francisco de Paula Santander, Ocaña, mostrado una ligera mejoría en el Plan de Estudios de Ingeniería Civil, seguido por la Ingeniería Mecánica y finalmente Sistemas, mostrando que existe una relación entre los niveles de exigencia de selección a la hora de ingresar a los estudios universitarios, la demanda estudiantil o deseo por ingresa a estudiar es mayor para la Ingeniería Civil, seguida por Mecánica, y en menor proporción Sistemas, por lo que hace necesario que la alta Dirección planté estrategias que le permita solucionar la problemática presentada, asegurando la calidad por espacios físicos y recurso humano.

Los resultados descritos en este estudio, llevan a establecer unos cuestionamientos en los cuales se debe resaltar la relación de los nuevos estilos de aprendizajes a las nuevas tendencias de estudio, utilizadas por los estudiantes, los cuales difieren de los hábitos de estudio, tradicionalmente reconocidos, como los relacionados por (Pozar, 2014) a través del cual se orienta el inventario de hábitos de estudio, el cual tiene como finalidad la evaluación de los hábitos de estudio que influyen en las tareas de aprendizaje en cuanto a cuatro aspectos fundamentales: las condiciones ambientales del estudio, la planificación del estudio, la utilización de materiales y la asimilación de contenidos.; ya que en la actualidad, los estudiantes no necesariamente deben estudiar en ambientes controlados, muy bien iluminados, sin ruidos, sin que esto les pueda asegurar ser lo más conveniente para realizar dichos estudios en la actualidad, ya que se debe tener en cuenta que los estudiantes cuentan en su gran mayoría con índices de atención dispersos y esto les permite desarrollar sus estudios en ambientes con diferentes estímulos en especial tecnológicos como lo son la música, el WhatsApp, el internet entre otros.

\section{ANÁLISIS DE RESULTADO}

En la presente investigación los hábitos de estudió no se correlacionaron con el rendimiento académico, lo que significa que para tener buen rendimiento no necesariamente se debe tener buenos hábitos de estudio, ratificando lo afirmado por las investigaciones de Villegas y Muñoz (2009); Picasso, Villanelo y Lorenzo (2015); De la Peña (2015), situación que es contraria a lo manifestado por los autores: Pozar (2014), Martínez y Torres (2009), Ríos y Ramos (2013) quienes concluyeron en su momento que el bajo rendimiento se debe en general al desconocimiento de un adecuado manejo de métodos y técnicas de estudio, a su vez consideraron que los hábitos de estudio inciden positivamente en el rendimiento académico y llegaron a establecer la correlación entre las dos variables. Las cosas hoy en día parecen cambiar ya que desde hace unos pocos años la tecnología ha entrado a hacer parte del diario vivir, los jóvenes ya disponen te dispositivos conectados a redes sociales como Facebook o WhatsApp que sin duda están causando cambios en los hábitos de ellos, más aún pueden afectar positivamente o negativamente el estudio dependiendo del buen o mal uso que se le dé al dispositivo. Lo importante es desarrollar esas habilidades en los estudiantes para que el docente a través de su disciplina potencie las acciones que contribuyan al fortalecimiento de las habilidades adecuadas .

El resultado de aplicar el Inventario de Hábitos de Estudio de Pozar (2014), evidenció que los estudiantes de la Facultad de Ingeniería presentan poco o casi nada de hábitos de estudio, al igual que lo manifestado por Villegas y Muñoz (2009), quien en su investigación confirmó que los estudiantes deben corregir sus hábitos para lograr terminar su formación profesional con éxito, de igual forma se debe considerar lo manifestado por Nuñez y Sanchez (1991), quienes determinaron que existen otros factores que intervienen en el estudio: Aspectos como la personalidad, motivación y aptitudes e intereses, que deben considerase, ya que pueden llegar a afectaer significativamente el estudio. Del mismo modo, se debe tener en cuenta que los tiempos han cambiado, hoy en día, los contextos se acceleran cada vez más, donde la tecnología juega un papel protagonico, de ahí la importancia de considerar como interviene en el aprendizaje para aprovecharla, los resultados 
obtenidos en la investigación, muestran que existe una gran oportunidad en cuanto a propuestas pedagógicas encaminadas a desarrollar los hábitos de estudio para mejorar el rendimiento académico en la Universidad Francisco de Paula Santander, seccional Ocaña.

\section{CONCLUSIONES}

El instrumento de preguntas abiertas permitió caracterizar los hábitos de estudio en la Facultad de Ingeniería, mostrando que los estudiantes prefieren el hábito de la lectura por encima de otros, aunque presentan una multitud de gustos a la hora de definir las estrategias efectivas para el estudio, entre las que se encuentran: repasar, practicar, tienen claro la disposición que deben tener hacia el estudio, les gusta hacer mapas mentales, dicen ser analíticos, tratan de concentrase (para ello, buscan contar con un ambiente limpio que no les genere distracciones) por lo general, estudian en la habitación, prefieren estudiar solos, aunque en algunas ocasiones lo hacen acompañados, tiene claro la importancia del estudio y que los motiva llegar a ser profesionales competentes. Resultados que son contradictorios a los presentados por LASSI (Weinstein et al, 1987) quienes encontraron que los estudiantes presentan un nivel bajo en la actitud, motivación, ansiedad y autoevaluación ante el estudio, caso contrario a (Picasso et al. 2015) quienes manifestaron que los estudiantes leen solo en forma ocasional, hábito que fue inculcado desde la niñez o en el colegio y que les gusta hacer solo en forma ocasional, acuden a la lectura durante viajes o cuando se encuentran en casa, los temas que más les leer gusta están relacionado con los hobbis, la ficción y textos complementarios al estudio, lo que quiere decir que los docentes deben seguir trabajando para inculcar el hábito de la lectura en los estudiantes ya que es esencial para el desarrollo de los demás hábitos.

\section{REFERENCIAS}

Aguilar, Flórez, M., \& Gómez, Á. (s.f.). Los estilos de Aprendizaje en los Estudiantes del Semillero de Fisica de la Uis.

Álvarez, M., \& Fernández, R. (2013). CHTE. Cuestionario de Hábitos y Técnicas de Estudio. Madrid: TEA Ediciones.

Andes, U. d. (2014). Determiantes de la Deserción. Bogotá .

Caballero Amaury, Velasco Gabriel, Pardo García A. (2013). Differentiations of objects in diffuse databases. Revista colombiana de tecnologías de Avanzada. 2 (22). Pág. 131 - 137.

Cartagena, M. (2008). Relación entre la autoeficacia y el rendimiento Escolar y los Hábitos de Esudio en alumnos de Secundaria. Revista Iberoamerica sobre Calidad, Eficacia y Cambio en Educación, 65.

Castellanos, S., Palacio, M. E., Cuesta, M., \& García, E. (2011). Cuestionario de Evaluación del Procesamiento Estratégico de la Información para Universitarios (CPEI-U). Revista Electrónica de Metodología Aplicada, 16.

Covey, S. R. (1997). Los 7 hábitos de la Gente Altamente. Buenos Aires: Paidos.

De la Peña, B. (2015). El rendimiento Académico en Alumnos de Enfermería y su relación con hábitos de Estudio y Estilos de Aprendizaje. Educación , 61.

Ebee, L. (2014). Cómo Aprender a Estudiar. Alcobendas: Libsa.

Escalante, L. E., Escalante, Y. I., Linzaga, C., \& Merlos, M. E. (2008). Comportamiento de los estudiantes en función a sus hábitos de estudio. Electrónica "Actualidades Investigativas en Educación”, 1-15.

Fabiola Cruz Núñez, A. Q. ( 2011). Hábitos de Estudio y Rendimiento Académico. Actualidades Investigativas en Educación, 3.

Francisco Camarero Suárez, F. M. (2000). Estilos y estrategias de aprendizaje en estudiantes universitarios. Psicothema, 615-622.

Gallego, O. M. (2010). Caracteristicas de los Hábitos de Estudio, la Ansiedad y la Depresión en Estudiantes de Psicologia . Revista Iberoamerica de Psicología, 57.

Gross, E. L. (2014). Cómo aprender a Estudiar . Madrid : Libsa.

Guzmán, C., Durán, D., \& Franco, J. (2009). Deserción Estudiantil. Bogotá: Imprenta Nacional de Colombia.

Hernández, C., Rodrígue, N., \& Ángel, V. (2012). Los hábitos de Estudio y Motivación pra el Aprenidizaje de los Alumnos en Tres Carrerar de Ingeniería . Revista de la Educación Superior, 71.

Hernández, F. (1988). Metódos y Técnicas de Estudio en la Universidad. Bogotá: Presencia Ltda.

Hernandez, F. (2001). Metodología del Estudio . Bogotá: McGraw Hill.

Hernández, F. (2001). Metodología del Estudio . Bogotá: McGRAW- Hill.

Hernandez, H. P., \& García, G. L. (s.f.). Enfoques, métodos y procedimeintos en la psicología del estudio . 
Martínez, O. (1997). Los adolescentes ante el estudio. España: Omagraf, S.L.

Mori, M. d. (2012). Deserción Universitaria en Estudiantes de una Univerisdad Privada de Iquitos. Revista Digital de Investigación en Docencia Universitaria, 66.

Muñoz-Repiso, Tejedor, F., \& García-Valcárcel, A. (2007). Causas del bajo rendimiento del estudiante universitario. de Educación, 443473.

Nunez, C., \& Sanchez, J. C. (1991). Hábitos de estudio y rendimiento en EGB y BUP. Un estudio comparativo. Complutense de Educación, 43.

Ortega, B. (2012). Hábitos de Estudio y Rendimiento Académico en Estudiantes de Segundo de Secundaria de una Institución Educativa del Callao. En B. Ortega, Académico en Estudiantes de Segundo de Secundaria de una Institución Educativa del Callao. Lima: Universidad San Ignacio de Loyola.

Picasso, M., Villanelo, M., \& Lorenzo, B. (2015). Hábitos de Lectura y Estudio y su Relación con el Rendimiento Académico en Estudiantes de Odontología de una Unversidad Peruana. Kiru, 19.

Pozar, F. (2014). Inventario de Hábitos de Estudio. Madrid: Tea.

Rincón, I., Suarez, S. A., \& Sánchez, E. (2015). Pensamiento crítico de los estudiantes que utilizan pensamiento crítico de los estudiantes que utilizan. Revista Colombiana de Tecnologías de Avanzada, 133.

Ríos, V., \& Ramos, D. (2013). Hábitos de Estudio y Rendimiento Académico en los Estudiantes de Carreras Profesionales de Ingeniería, Ecoturismo y Educación de la Universidad Nacional Amazónica . Educación.

Sampieri, H., Fernandez, C., \& Baptista, P. (2006). Metodología de la Investigación. México D.F: McGraw-Hili.

Torres, M., Tolosa, I., Urrea, C., \& Monsalve, A. (2009). Inventario de hábitos de estudio en una clase para toma. Bogotá: Cienc. Salud.

Torres, M., Tolosa, I., Urrea, M. d., \& Monsalve, A. M. (2009). Hábitos de estudio vs fracaso académico. Universidad de Costa Rica, 15-24.

Valentín Martínez, O. P., \& Barberis, L. T. (2013). Ansiedad en Estudiantes Universitarios: estudio de una Muestra de Alumnos de la Facultad De Educación. Uclm.

Valenzuela, J., \& Flores, M. (2012). Fundamentos de investigación educativa, Volumnen 2. Mexico .

Villarroel, C. (1989). Evaluación y conducción de los aprendizajes. Mexico : Trillas.
Villegas, C. A., \& Muñoz, F. (2009). Hábitos de estudio de los alumnos en el área de Química Orgánica y su impacto en el rendimiento académico. Biotencia.

Villota, M. F. (2013). Hábitos y técnicas de estudio en la Universidad Mariana. Unimar, 81-97. 